\title{
Antibody Response to ChAdOxl-nCoV-I 9 Vaccine Among Recipients in Bangladesh: A Prospective Observational Study
}

\author{
Ashraful Hoque (D) \\ Anindita Das Barshan ${ }^{2}$ \\ Forhad Uddin Hasan \\ Chowdhury ${ }^{2}$ \\ Jannatul Fardous ${ }^{3}$ \\ Mohammad Jahid Hasan (iD ${ }^{3}$ \\ Md Abdullah Saeed Khan (iD) ${ }^{3}$ \\ Ahmedul Kabir ${ }^{4}$ \\ 'Department of Blood Transfusion, \\ Sheikh Hasina National Institute of Burn \\ and Plastic Surgery, Dhaka, Bangladesh; \\ ${ }^{2}$ Department of Medicine, Dhaka Medical \\ College Hospital, Dhaka, Bangladesh; \\ ${ }^{3}$ Tropical Medicine and Infectious Disease \\ Division, Pi Research Consultancy \\ Center, Dhaka, Bangladesh; ${ }^{4}$ Department \\ of Medicine, Mugda Medical College \\ Hospital, Dhaka, Bangladesh
}

Correspondence: Mohammad Jahid Hasan Email dr.jahid6I@gmail.com; jahid@pircc.org
Purpose: The aim of the study was to assess the antibody response to the ChAdOx1-nCoV vaccine in individuals who were not previously infected by COVID-19.

Patients and Methods: All people aged 18-65 years who received their first vaccination with ChAdOx1-nCoV from March to May 2021 were approached for inclusion. Individuals with sufficient antibody titers against SARS-CoV-2 infection before vaccination were considered previously infected and were excluded from the analysis. We observed viral spike protein RBD-S1-specific IgG antibody levels at day 28 of the first dose of vaccination and day 14 of the second dose of vaccination (74 days from index vaccination). An optical density ratio (ODR) of $>1.1$ was considered to have a positive antibody response, 0.8 to 1.1 borderline and $<0.8$ was denoted as negative. Informed consent was ensured before enrollment, and ethical principles conformed with the current Declaration of Helsinki.

Results: This observational study comprised 769 infection-naïve individuals (mean age 40.5 years, 38.9\% female). Spike-specific IgG antibody responses elicited after the first and second doses of vaccine were $99.9 \%$ and $100 \%$, respectively. The median ODR was 5.43 (interquartile range [IQR]: 4.32-6.98) and 10.90 (IQR 9.02-11.90) after the first and second doses. Higher age was associated with lower antibody levels after both dosages. However, no sex-specific variation was seen. People with comorbidity had a lower antibody level after the second dose. Tenderness (51.46\%) and fever $(19.30 \%)$ were the most common local and systemic side effects after vaccination.

Conclusion: This study was one of the earlier attempts in the country to assess the antibody response to ChAdOx1-nCoV vaccine recipients. The results imply that general people should be encouraged to take the vaccine at their earliest.

Keywords: ChAdOx1-nCoV, vaccine, COVID-19, Bangladesh, antibody response

\section{Background}

Since its first report from Wuhan, China, ${ }^{1}$ coronavirus disease 2019 (COVID-19) has claimed millions of lives around the world. ${ }^{2}$ It has significantly affected people's lives with substantial disruption of health services and considerable impact on the global economy. Currently, the most promising hope for ending this pandemic is the successful rollout of widespread COVID-19 vaccination.

Scientists focused on the development of COVID-19 vaccine candidates shortly after the virus was characterized. To date, 184 and 105 vaccine candidates are in the preclinical and clinical development phases, respectively. ${ }^{3}$ A total of 17 vaccines are currently being offered to the public, with 8 of them being actively monitored. 
Although many vaccines have been approved for public use, a significant hurdle is to mass produce and distribute the vaccine to vast populations who require it. Governments in low-resource countries struggle to obtain enough vaccines given the high costs, human resource constraints, and differences in vaccine efficacy in producing immunity against severe acute respiratory syndrome coronavirus 2 (SARS-CoV-2). ${ }^{4}$ Several policies have recently been proposed to minimize vaccine shortages, such as delaying the second dose for everyone or prioritizing vaccinations for infection-naïve people. As a result, in addition to prioritization of high-risk groups and diplomacy for low-cost sources, the efficacy of the currently approved vaccines in persons without prior COVID-19 infection is an active area of research. ${ }^{5}$ Additionally, some reports suggest that mixing and matching COVID19 vaccines might produce a robust immune response. ${ }^{6}$

Oxford AstraZeneca (ChAdOx1-nCoV) vaccine was shown to produce robust antibody response against SARS CoV-2 with a good safety profile in all age groups after a booster dose (second dose) in Phase 2/3 clinical trials. ${ }^{7,8}$ Real-world evidence from people vaccinated with ChAdOx1-nCoV demonstrated a marked reduction in hospitalization and deaths, and a reduction in the likelihood of symptomatic disease and household transmission of the virus. $^{9}$

The Government of the People's Republic of Bangladesh managed to obtain the Oxford AstraZeneca (ChAdOx1-nCoV) vaccine initially and began mass vaccination by 7 th February 2021. The country has successfully completed more than one million dosages of the vaccine as of 28th June 2021. ${ }^{10}$ However, the immunogenicity induced by the vaccine has not been explored in the country. Therefore, the aim of the present study was to assess the antibody response to the ChAdOx $1-n C o V$ vaccine among vaccine recipients in Bangladesh.

\section{Materials and Methods}

\section{Study Place, Population and Participant Selection}

The observational study was carried out at the Department of Transfusion Medicine, Sheikh Hasina National Institute of Burn and Plastic Surgery, Dhaka, Bangladesh. This tertiary care setting was designated as a dedicated COVID-19 unit by the government during the upsurge of COVID-19 cases in the country. The study period spanned from March 1, 2021 to May 31, 2021. All people aged 18-
65 years who registered for vaccination using the central online vaccine registration system and arrived at the vaccination site to receive the first dose of ChAdOx1-nCoV -19 vaccine were approached. We excluded vaccine recipients younger than 18 years and older than 65 years who had any kind of autoimmune disease and/or immunosuppressive conditions. Baseline antibody assessment was performed for vaccine recipients. Individuals with sufficient antibody titers [optical density ratio (ODR) of $>1.1$ ] against SARS-CoV-2 infection before vaccination were considered previously infected and were excluded. Individuals with baseline antibody titers lower than $<1.1$ were considered negative for antibody against the virus and were considered for inclusion. A total of 912 people were approached, and 855 participants were initially selected for inclusion (Supplementary Figure 1). All participants were given two doses of ChAdOx1 $\mathrm{nCoV}-19$ at a dose of $5 \times 10^{10}$ viral particles (standard dose) 60 days apart. We observed viral spike protein RBD-S1-specific IgG antibody levels at day 28 of the first dose of vaccination and day 14 of the second dose of vaccination (74 days apart from the index vaccination). All participants completed a full vaccination schedule, and no vaccine breakthrough cases were reported. A detailed road map of the study is illustrated in Figure 1.

\section{Measures}

To obtain data, we used a pretested structured questionnaire covering demographic details and personal history with comorbidities. The questionnaire was prepared based on the consensus of the investigators after reviewing relevant published literature and the best possible insight of the author's team. Baseline assessments included a review of inclusion and exclusion criteria, medical history, vital sign measurement, history-directed clinical examination, and collection of serum for SARS-CoV-2 serology. With all aseptic precautions, approximately $2 \mathrm{~mL}$ of venous blood was drawn by the two trained nurses from the antecubital vein for baseline antibody assessment. The samples were then stored in a collection tube, and plasma were separated by centrifugation (15 minutes at 2200 RPM). Plasma aliquots were coded, stored at $-20^{\circ} \mathrm{C}$ and assayed blindly. Serological testing for antibodies to the RBD of the S1 subunit of the viral spike protein $(\operatorname{IgG})$ was performed using an anti-SARS-CoV-2 enzyme-linked immunosorbent assay (ELISA) from EUROIMMUN as per the instructions supplied by the manufacturer. ${ }^{11}$ In short, the first $10 \mu \mathrm{L}$ plasma samples were diluted to 


\section{Road map of the study}

(March 1, 2021-May 31, 2021)

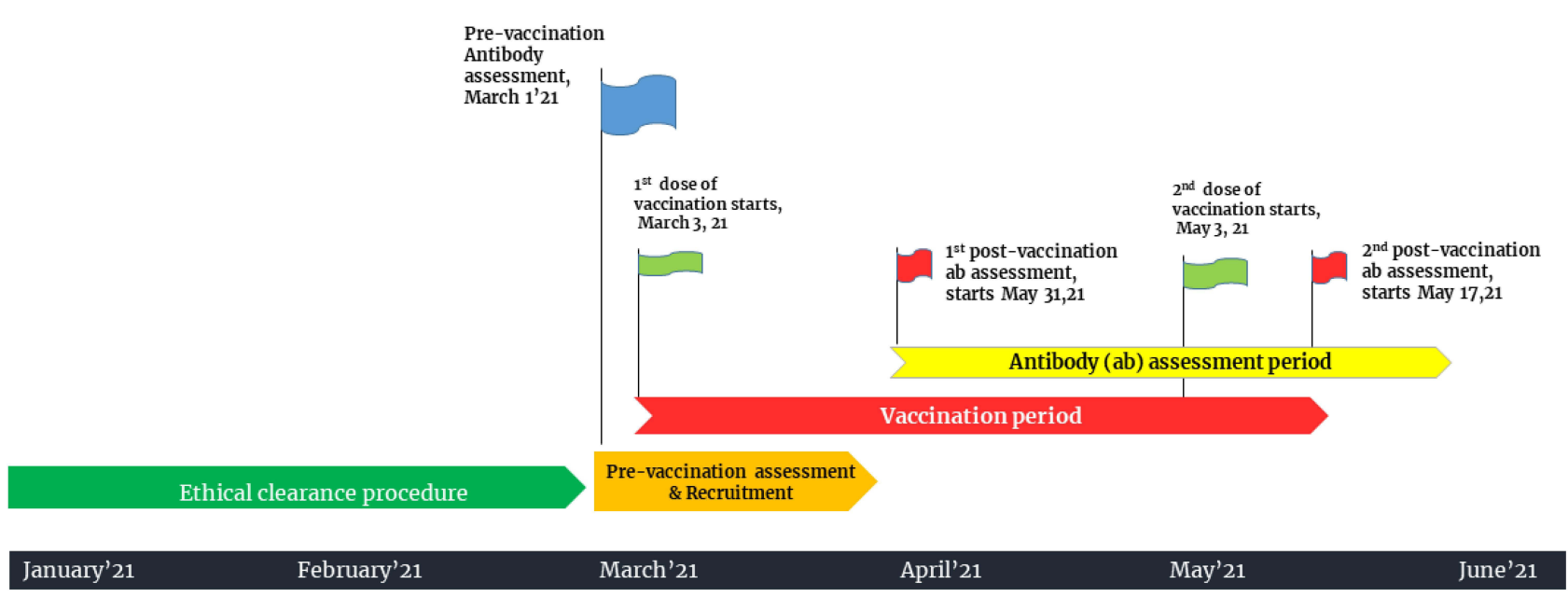

Figure I Road map of the study.

$1: 101$ and then incubated in the wells with anti-SARSCoV-2 ELISA IgG kits (Euroimmun, Lubeck, Germany). A second cycle of incubation using an enzyme-labeled anti-human IgG antibody (supplied with the kit) was then performed. Then, a specific chromogen solution was added to the well, which produced a quantifiable change in color, the intensity of which was proportional to the antibody present in the plasma. Semiquantitative results were evaluated by calculating the ratio of extinction at $450 \mathrm{~nm}$ (optical density ratio [ODR]) of each sample over the calibrator. As per the instructions, a cutoff ratio of ODR value of $>1.1$ was considered a positive test for SARS COV-2 IgG, 0.8 to 1.1 as borderline and $<0.8$ as negative. In this study, a borderline cutoff ratio of 0.8 to 1.1 was outlined as a negative result. ${ }^{11}$

Following confirmation of the level of the antibody and consent procedure, the vaccine recipient was advised to take the first dose of ChAdOx1 nCoV-19 (AZD1222) vaccine. The recombinant ChAdOx1 nCoV-19 (COVISHIELD ${ }^{\mathrm{TM}}$ ) was manufactured by Serum Institute of India Pvt Ltd, Lot no-4120Z008 and 4120Z024. Before mass vaccination, the vaccine schedule was also approved by the regulatory agency of the Bangladesh government. Proper cold chain was maintained according to the manufacturer's instructions and was ensured by the vigilant team assigned to vaccine transfer. The vaccine was provided intramuscularly in the right/left deltoid region by the senior staff nurse. Antibody responses elicited by the first and second doses of vaccine were performed at day 28 and day 74 of index vaccination (14 days apart from the second dose of vaccination) by following similar procedures as previously described. Protocol deviations were not permitted during recruitment, and the first dose of vaccination but flexibility was allowed if necessary: on day $60 \pm 2$ of the second dose of vaccination and on days $28 \pm 2$ and $74 \pm 4$ of post-vaccination antibody assessment. All records were kept with strict confidence.

Participants were instructed to contact the study physician immediately if they experienced any symptoms associated with COVID-19 vaccines for a period of 14 days after completion of the second dose. They also received a regular reminder to do so over the phone. The protocol kept options for them to perform a nucleic acid amplification test (NAAT) against SARS-CoV-2 lest any signs of COVID-19 occur. However, we have dealt with no such case during the follow-up period.

\section{Ethical Consideration}

The study protocol was approved by the Ethical Review Committee of Sheikh Hasina National Institute of Burn \& Plastic Surgery [Memo No- SHNIBPS/Feb (20)/05]. Informed written consent was obtained from the participants before inclusion, and ethical principles conformed to the current Declaration of Helsinki. In addition, the study was conducted according to the ethical and legal requirements of the country. 


\section{Statistical Analysis}

Participants' demographic characteristics and comorbidity profiles were expressed as proportions. KolmogorovSmirnov test and histogram with normal curves were used to check the normality of the data. Antibody level was expressed as median and interquartile range (IQR) and demonstrated using Boxplots with jitter. Median antibody levels were compared across groups using the Mann-Whitney $U$-test, and across time using Friedman's ANOVA. Pairwise comparisons between time points were carried out using Wilcoxon's signed rank test. A mixed model ANOVA was also carried out to assess the effect of age, sex and comorbidity on antibody response at three time points. As Mauchly's test of the sphericity assumptions was violated, Huyan-Field test was used to report the significance of the main and interaction effects. Bonferroni adjustments had been used for simple main effect analysis by post-hoc pairwise comparisons when there was significant interaction. Scatterplots were constructed between age and antibody levels. Statistical packages SPSS Version 26 and R Studio version 1.4.1106 were used for statistical analysis and graph building.

\section{Results}

Between March 1 and May 31, 2021, 769 infection-naïve participants were recruited and vaccinated with two doses of ChAdOx1 nCoV-19. The mean age of the participants was 40.5 (10.3) years (SD), 38.9\% were female. Of all, $36.7 \%$ had at least one comorbidity, such as hypertension, diabetes mellitus, bronchial asthma or chronic obstructive pulmonary disease (COPD). The baseline characteristics of the participants are shown in Table 1.

Post vaccination spike-specific IgG antibody-level responses elicited by a single vaccine dose was $99.9 \%$ $(\mathrm{n}=768)$, and by two dosages was $100 \%(\mathrm{n}=769)$. The median antibody level measured in optical density ratio (ODR) at baseline (pre-vaccination), after the first dose and after the second dose was 0.69 (IQR 0.43-0.92), 5.43
Table I Characteristics of Study Participants $(n=769)$

\begin{tabular}{|l|l|}
\hline Variable & $\mathbf{n}(\%)$ \\
\hline Age (years), mean (SD) & $40.5(10.3)$ \\
\hline Age groups (years) & \\
$21-30$ & $100(13.0)$ \\
$31-40$ & $352(45.8)$ \\
$41-50$ & $205(26.7)$ \\
$51-60$ & $72(9.4)$ \\
$>60$ & $40(5.2)$ \\
\hline Sex & \\
Female & $299(38.9)$ \\
Male & $470(61.1)$ \\
\hline Comorbidities & \\
Hypertension & $278(36.2)$ \\
Diabetes & $39(5.1)$ \\
Bronchial Asthma/COPD & $6(0.8)$ \\
Any comorbidity & $282(36.7)$ \\
\hline
\end{tabular}

Notes: $p$-value determined by Independent Samples $t$ Test, Chi-square Test and Fisher's Exact Test where appropriate.

(IQR 4.32-6.98) and 10.90 (9.02-11.90), respectively (Table 2). The antibody levels at baseline, postvaccination dose 1 and postvaccination dose 2 among vaccine recipients are illustrated in Figure 2.

Antibody levels were compared among the vaccine recipients with respect to age, sex (male and female) and the presence or absence of any comorbidity at all-time points (Table 3). Both sexes had a similar antibody level at all time points. However, participants with comorbidities had a significantly lower antibody response after the second dose of vaccine $(p=0.01)$. Participants aged $>55$ years had a significantly lower antibody level than patients age spanning $18-55$ years $(\mathrm{p}<0.001)$ in both first and second doses of the vaccination. Additionally, a higher age was associated with a lower antibody response following each dose of the vaccination (Figure 3). A mixed model ANOVA revealed a significant interaction between age group $(p<0.001)$ and

Table 2 Antibody Response of the Vaccine Recipient at Pre-Vaccination, Post-Vaccination Dose I and Post-Vaccination Dose 2

\begin{tabular}{|l|l|l|l|l|}
\hline Antibody Response Measured by ODR & Pre-Vaccination & Post-Vaccination D-I & Post-Vaccination D-2 & $\mathbf{p}$-value \\
\hline Median (IQR) & $0.69(0.43-0.92)$ & $5.43(4.32-6.98)^{\mathrm{a}}$ & $10.90(9.02-11.90)^{\mathrm{a}, \mathrm{b}}$ & $<0.00 \mathrm{I}^{*}$ \\
Response category & $\mathrm{n}(\%)$ & $\mathrm{n}(\%)$ & $\mathrm{n}(\%)$ \\
Negative (ODR <0.8) & $496(64.5)$ & $\mathrm{I}(0.10)$ & - & - \\
Borderline (ODR 0.8-I.I) & $273(35.5)$ & - & $769(100.00)$ \\
Positive (ODR $>$ I.I) & - & $768(99.9)$ & \\
\hline
\end{tabular}

Notes: *Friedman's two-way analysis of variance (ANOVA) with post-hoc analysis using Wilcoxon signed rank test and Bonferroni adjustments $\left({ }^{\mathrm{a}} \mathrm{p}<0.00 \mathrm{I}\right.$ compared to prevaccination, ${ }^{b} p<0.001$ compared to post-vaccination $\left.D-I\right)$.

Abbreviations: D-I, Dose I; D-2, Dose 2; ODR, optical density ratio; IQR, interquartile Range. 


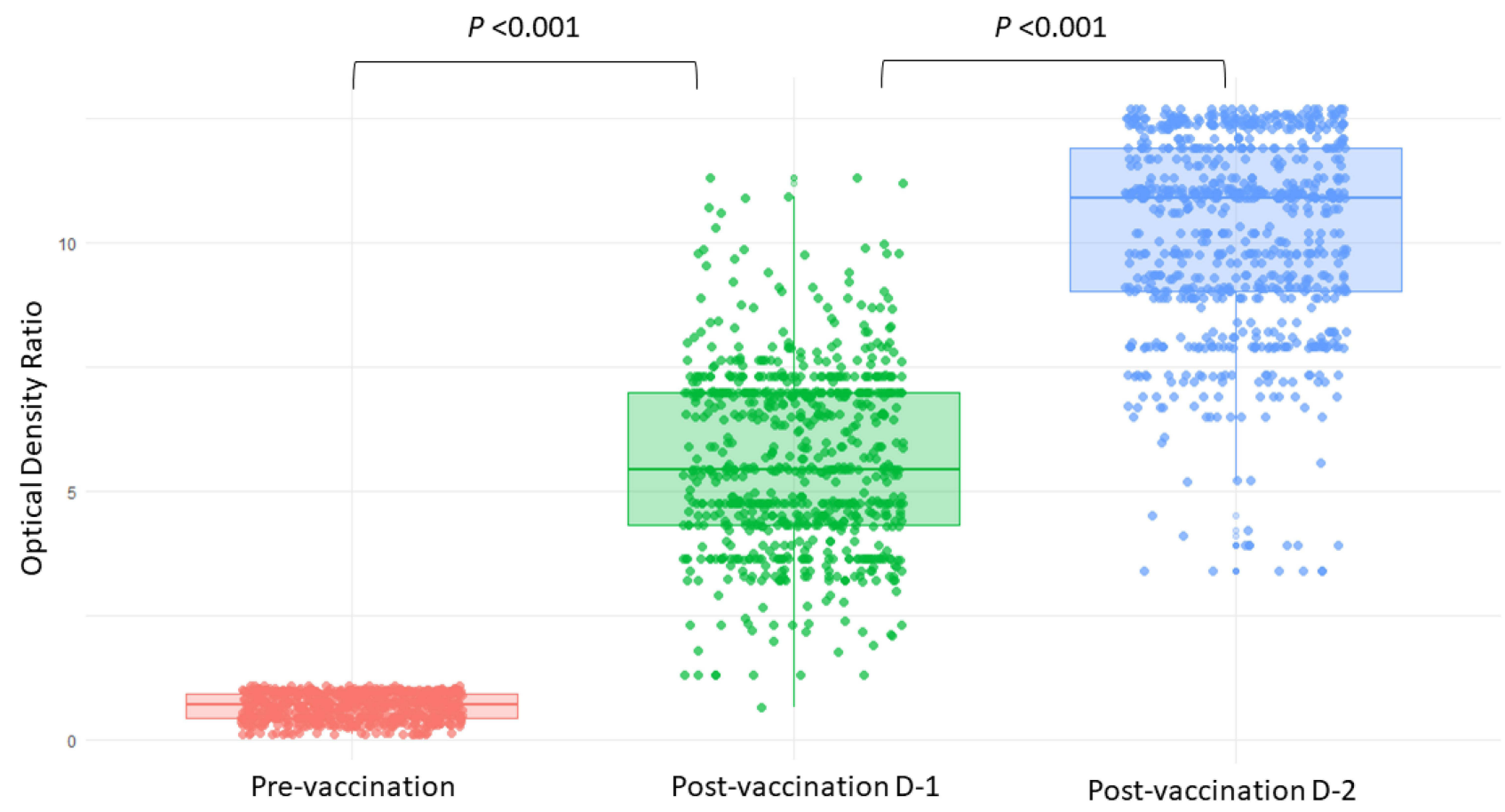

Figure 2 jitter-box plots showing antibody levels at pre-vaccination, post-vaccination dose $I$ and post-vaccination dose 2 among participants. $P$-value was determined by Wilcoxon Signed Rank test. $P<0.00$ I between (i) pre-vaccination and post-vaccination dose-I, and between (ii) post-vaccination dose-I and post-vaccination dose-2. Person without infection had Optical Density Ratio (ODR) below 0.8 at pre-vaccination period hence a smoothened appearance.

Abbreviations: D-I, Dose I; D-2, Dose 2.

comorbidity $(\mathrm{p}=0.003)$ with repeated measures of antibody response at three points in time, indicating that the antibody response was different between age groups (18-55 years and $>55$ years) and comorbidity groups (comorbidity present and comorbidity absent) across time. However, the interaction of comorbidity with time was significant only after the second dose of vaccine $(p=0.003)$. This means that the antibody response among comorbid individuals was statistically similar to that of those without comorbidity after the prime dose of ChAdOX nCoV-19, but were significantly lower than those without comorbidity after the booster dose of vaccine. On the other hand, patients' sex did not show significant interaction with time, implying that there were no sexrelated differences in antibody response after any dose of the vaccine (Table 4). Figure 4 depicts the changes in the estimated marginal means of antibody response among different groups of people at three time points.

The most common local adverse event at the site of injection was tenderness $(51.46 \%)$ followed by warmth (14.8\%), swelling (5.26\%) itching (3.51\%), and redness $(2.92 \%)$. Fever was the most common systemic adverse event $(19.3 \%)$ followed by diarrhea $(0.35 \%)$ (Table 5$)$. However, no breakthrough cases were reported during the period of 14 days after the second dose.

\section{Discussion}

The COVID-19 pandemic is causing incessant disruption in people's activities and taking millions of lives worldwide. Globally, just over 300 million vaccine doses had been administered as of March 2021. This figure gives us the hope that life without a mask might return someday. However, COVID-19 vaccination is facing several obstacles that could jeopardize its success. These include large-scale production efforts, new virus strains that are potentially dangerous, and public skepticism about vaccines. However, reports about the effectiveness and safety, if communicated properly, can abate the fear surrounding it. Immunogenicity responses among people after vaccination against COVID-19 in Bangladesh have not been reported yet. We aimed to study the immunogenicity of a viral vector-based COVISHIELD ${ }^{\mathrm{TM}}$ (ChAdOx1 nCoV-19-recombinant) vaccine in producing an antibody response in the Bangladeshi population who were not infected COVID-19.

We found that every participant except one in our cohort who received the first dose of vaccine developed a significant antibody response. The response was a cent percent after the second dose. This is consistent with findings from Phase $1 / 2$ and 2/3 trials of ChAdOx1 
Table 3 Antibody Response of the Participants Categorized by Age, Sex and Comorbidities

\begin{tabular}{|c|c|c|c|}
\hline \multirow[t]{4}{*}{ Period } & \multicolumn{2}{|l|}{ Characteristics } & \multirow[t]{4}{*}{ P-value* } \\
\hline & \multicolumn{2}{|l|}{ Age Group (Years) } & \\
\hline & $18-55$ & $>55$ & \\
\hline & Median (IQR) & Median (IQR) & \\
\hline \multirow{7}{*}{$\begin{array}{l}\text { Pre-vaccination } \\
\text { Post-vaccination D-I } \\
\text { Post-vaccination D-2 } \\
\text { P-value** }\end{array}$} & $0.68(0.43-0.92)$ & $0.74(0.45-0.92)$ & \multirow{4}{*}{$\begin{array}{l}0.254 \\
<0.001 \\
<0.001\end{array}$} \\
\hline & $5.49(4.39-6.98)^{\mathrm{a}}$ & $4.76(3.29-6.80)^{\mathrm{a}}$ & \\
\hline & $10.90(9.10-11.90)^{\mathrm{a}, \mathrm{b}}$ & $9.10(6.90-11.30)^{a, b}$ & \\
\hline & $<0.001$ & $<0.001$ & \\
\hline & \multicolumn{2}{|l|}{ Sex } & \\
\hline & Female & Male & \\
\hline & Median (IQR) & Median (IQR) & \\
\hline \multirow{7}{*}{$\begin{array}{l}\text { Pre-vaccination } \\
\text { Post-vaccination D-I } \\
\text { Post-vaccination D-2 } \\
\text { P-value** }\end{array}$} & $0.72(0.43-0.92)$ & $0.68(0.43-0.92)$ & 0.866 \\
\hline & $5.43(4.39-6.98)^{\mathrm{a}}$ & $5.43(4.31-6.98)^{\mathrm{a}}$ & 0.753 \\
\hline & $10.70(9.02-\mid 1.90)^{\mathrm{a}, \mathrm{b}}$ & $10.90(9.02-11.90)^{\mathrm{a}, \mathrm{b}}$ & 0.433 \\
\hline & $<0.001$ & $<0.001$ & \\
\hline & \multicolumn{2}{|l|}{ Co-morbidities } & \\
\hline & Present & Absent & \\
\hline & Median (IQR) & Median (IQR) & \\
\hline Pre-vaccination & $0.72(0.43-0.92)$ & $0.68(0.43-0.92)$ & 0.763 \\
\hline Post-vaccination D-I & $5.43(4.30-6.98)^{\mathrm{a}}$ & $5.49(4.39-6.98)^{\mathrm{a}}$ & 0.392 \\
\hline Post-vaccination D-2 & $10.60(8.21-\mid 1.90)^{\mathrm{a}, \mathrm{b}}$ & $10.90(9.1-\mid I .90)^{\mathrm{a}, \mathrm{b}}$ & 0.010 \\
\hline$P$-value** & $<0.001$ & $<0.001$ & \\
\hline
\end{tabular}

Notes: Antibody response measured in optical density ratio (ODR); $p$-value was determined by *Mann-Whitney U-Test and **Friedman's two-way analysis of variance (ANOVA) with post-hoc analysis using Wilcoxon signed rank test and Bonferroni adjustments ( ${ }^{\mathrm{a}} p<0.001$ compared to pre-vaccination, ${ }^{\mathrm{b}} p<0.00 \mathrm{l}$ compared to postvaccination $D-1)$.

Abbreviations: D-I, Dose I; D-2, Dose 2.

nCoV-19 (AZD1222) ${ }^{12,13}$ Moreover, antibody levels were higher after the second dose (ie Prime booster) than that of the first dose (ie Prime dose) among participants as was reported in the fifth clinical trial of AZD1222. ${ }^{11}$ Previous studies have shown that anti-spike IgG levels correlate with neutralizing antibody titers for all age groups. ${ }^{7}$ Hence, although an assessment of neutralizing titer was not possible in this study, we can assume that the high antibody titer achieved at the end of the second dose among our participants would convey protection from COVID-19 as long as it remains in the blood. Noticeably, we did not get any breakthrough infection for 2 weeks after the completion of the second dose among vaccine recipients, indicating a possible protection from the virus, which we hope can extend up to 20 weeks at least, as earlier studies have suggested. ${ }^{8}$

Our findings showed that the antibody response of infection-naïve vaccine recipients was lower with increasing age. Older adults ( $>55$ years) were more likely to get a boost in immunity after the second dose; however, it still remained significantly lower than that of younger recipients. This is in contrast to the results of the preliminary reports of the first clinical trial conducted using AZD1222 where such differences in response were not reported. ${ }^{8,12}$ But subsequent trials (phase 2/3) with the same vaccine showed an age-related decline in antibody response, ${ }^{7}$ which endorses our findings. Similar to our observations, two-dose mRNA vaccine trials have also got an absolute neutralizing antibody level lower in adults compared to younger adults. ${ }^{14}$ Additionally, we found no differences in antibody response across sex categories, which is consistent with their reports.

We noted that vaccine recipients with one or more comorbidities among HTN, DM, Bronchial Asthma and COPD showed a similar antibody response after the first dose to that of recipients without comorbidities. However, 

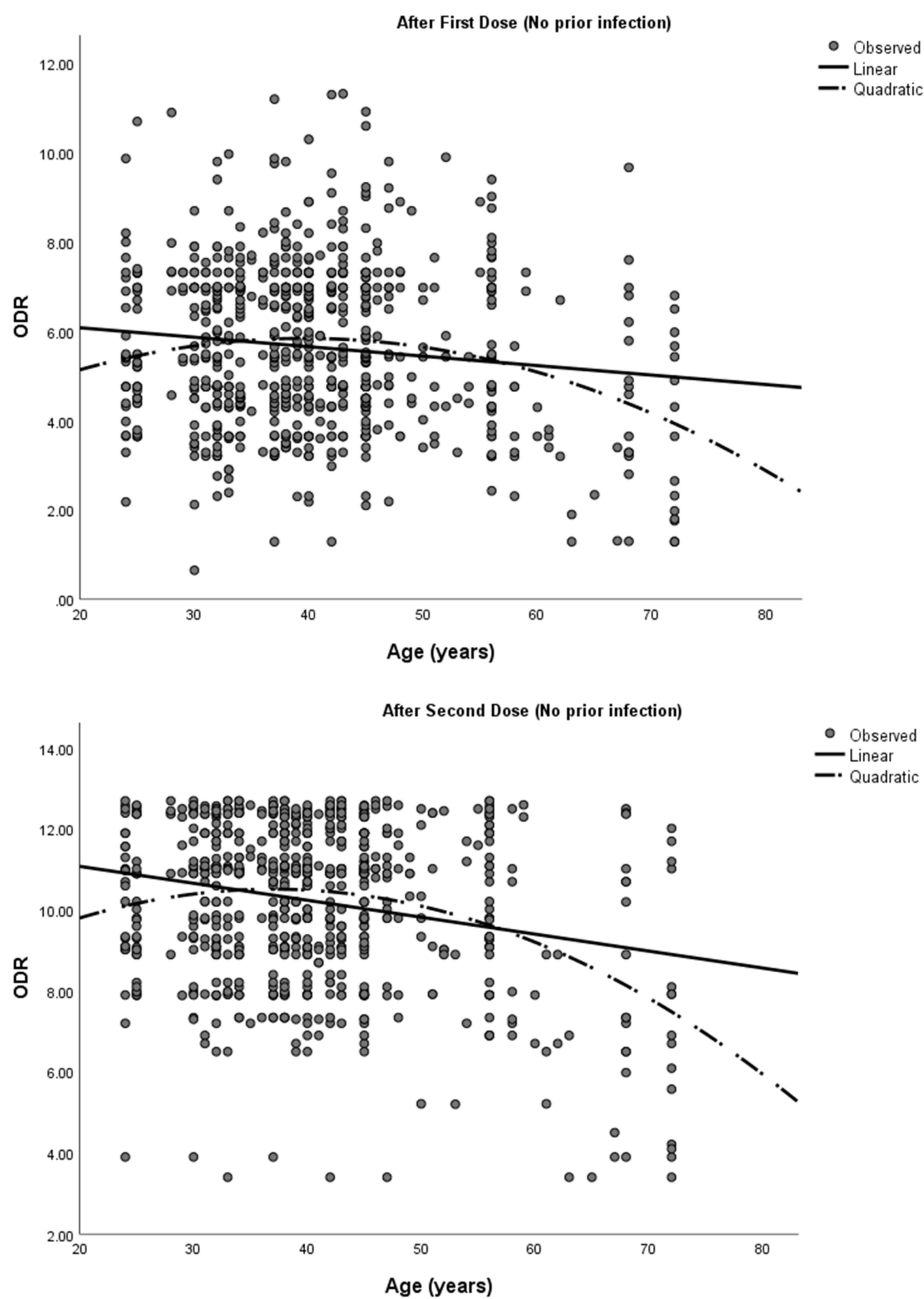

Figure 3 Scatterplots showing antibody response by age of the infection-naive participants. Abbreviations: ODR: Optical Density Ratio.

after the second dose, the antibody level failed to reach the same level in the former group compared to the latter group. Nonetheless, the antibody response was very high even among the participants with comorbidities indicating that the vaccine could give a good deal of protection in this subgroup of people. Most phase $2 / 3$ clinical trials with AZD1222 did not incorporate participants with comorbidities out of safety concerns. We might have to wait for the results of ongoing Phase 4 trials to find the pattern of response among comorbid individuals. However, as our study only included participants with controllable comorbid conditions, vaccines response among individuals with severe comorbidities cannot be inferred from this sample.

No serious adverse events like venous thromboembolic events following immunization were found in our 


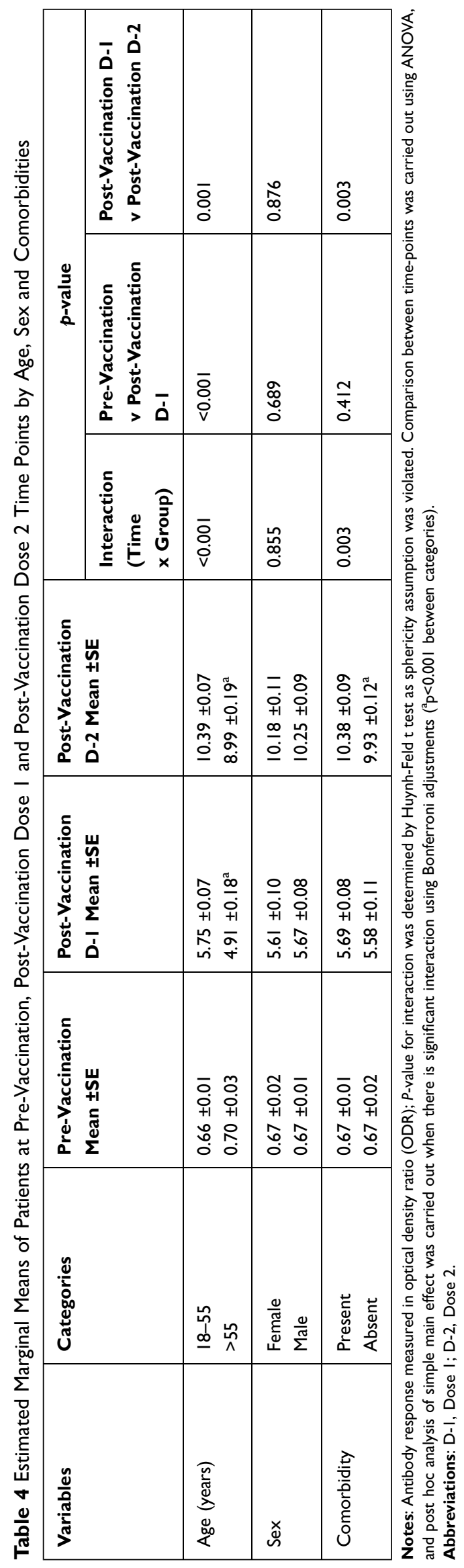

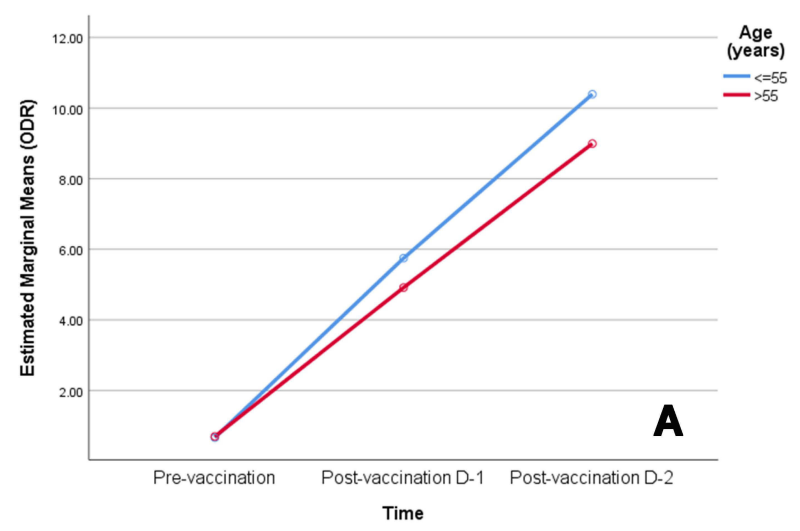
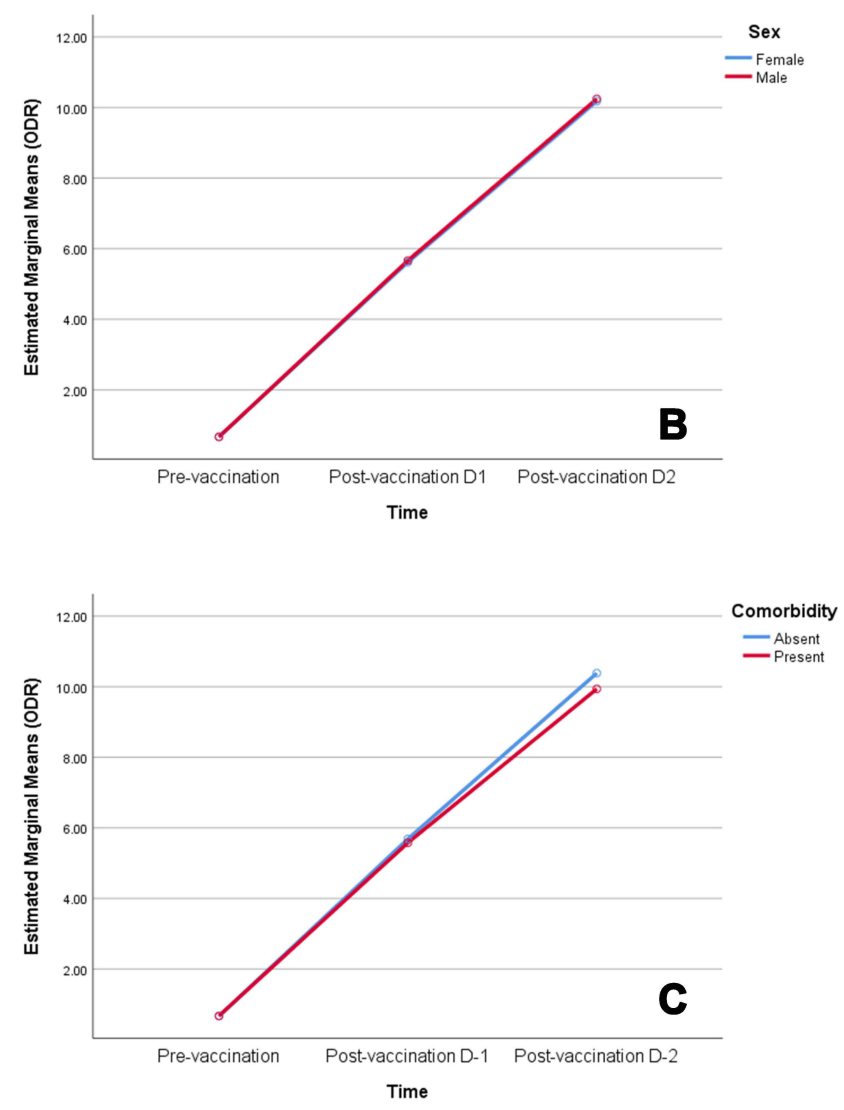

Figure 4 Profile plots of estimated marginal means of antibody response among participants by (A) age group, (B) sex and (C) comorbidity at pre-vaccination, postvaccination dose-I and post-vaccination dose-2 time points.

Abbreviation: ODR, optical density ratio.

participants within the 28 days follow-up after the first dose and 14 days follow-up after the second dose of the vaccine. When considering that a number of serious adverse events like cerebral venous thrombosis were very low in previous reports ${ }^{15}$ with a reported excess event of 2.5 (0.9 to 5.2) per 100,000 vaccinations, absence of serious events following immunization in our small sample is expected. Moreover, the mild local and 
Table 5 Side Effects After Completed Dosage of Vaccines Among Participants

\begin{tabular}{|l|l|l|}
\hline Side Effects & $\mathbf{n}$ & $\%$ \\
\hline Tenderness* & 396 & 51.46 \\
Fever & 148 & 19.30 \\
Warmth* & 114 & 14.85 \\
Swelling & 40 & 5.26 \\
Itching & 27 & $3.5 \mathrm{I}$ \\
Redness & 22 & 2.92 \\
Flu-like symptoms & 18 & 2.34 \\
Diarrhea & 3 & 0.35 \\
Vomiting & 0 & 0.00 \\
\hline
\end{tabular}

Note: *At the injection site.

constitutional symptoms reported immediately after the vaccine among our cohort, repeat the findings of previous studies and reassure against its safety concerns in the context of Bangladeshi people.

\section{Limitations of the Current Study}

Our study has several limitations. This was a single-center study, and we could not estimate the neutralizing antibody response among the vaccine recipients due to lack of facilities. In addition, we could not evaluate the longtime antibody response and safety profile among our participants. We acknowledge that the vaccine immunity is primarily cell-mediated immunity, which we were unable to evaluate. Hence, only the antibody titer studied in our research cannot guide the vaccine selection process.

\section{Recommendations}

Further, large sample multicenter phase 4 clinical trials with a longer duration of follow-up using ChAdOx1$\mathrm{nCoV}$ vaccine are recommended.

\section{Conclusion}

This study was one of the earliest attempts in Bangladesh to assess the antibody response of the ChAdOx-nCoV-19 vaccine among participants without baseline antibody levels. We found a robust immune response in vaccine recipients irrespective of infection status, age, sex and comorbidity. However, an age and comorbidity related lower antibody response was detected. These findings will be critical in making vaccination policies in the context of the country. Older people, particularly those with comorbidities, should be preferred in case of vaccine shortage. A third dose of vaccine for this group could be considered if adequate vaccines are available. The information regarding good antibody response among vaccine recipients in our study could help raise awareness about vaccination against COVID-19 among the general people of the country.

\section{Abbreviations}

COVID-19, Coronavirus disease 2019; ODR, Optical density ratio; RBD, Receptor binding domain; RT-PCR, Reverse transcriptase polymerase chain reaction.

\section{Data}

Available on request.

\section{Ethical Consideration}

Ethical measures were taken throughout the study period to maintain a high standard of confidentiality and anonymity of the participants. Formal ethical clearance was taken from the Ethical Review Committee of Sheikh Hasina National Institute of Burn \& Plastic Surgery (NoSHNIBPS/Feb (20)/05). Informed written consent was taken from the participants before inclusion.

\section{Consent of Publication}

All authors agreed to publish the article.

\section{Acknowledgments}

The author thanks the respondents who participated in the study during this stressful situation. The authors are also thankful to the research team of the 'Pi Research Consultancy Center' (www.pircc.org) for support throughout the study period, especially for journal selection and manuscript formatting.

\section{Author Contributions}

All authors made a significant contribution to the work reported, whether that is in the conception, study design, execution, acquisition of data, analysis and interpretation, or in all these areas; took part in drafting, revising or critically reviewing the article; gave final approval of the version to be published; have agreed on the journal to which the article has been submitted; and agree to be accountable for all aspects of the work.

\section{Funding}

This research received no external funding. 


\section{Disclosure}

The authors declare that there are no conflicts of interest in this work.

\section{References}

1. Zhou P, Yang X-L, Wang X-G, et al. A pneumonia outbreak associated with a new coronavirus of probable bat origin. Nature. 2020;579 (7798):270-273. doi:10.1038/s41586-020-2012-7

2. World Health Organization: WHO. The true death toll of COVID-19: estimating global excess mortality [Internet]; 2020. Available from: https://www.who.int/data/stories/the-true-death-toll-of-covid-19estimating-global-excess-mortality. Accessed November 16, 2021.

3. Gavi. The COVID-19 vaccine race - weekly update [Internet]; 2021. Available from: https://www.gavi.org/vaccineswork/covid-19-vaccinerace. Accessed November 16, 2021.

4. Choi EM. COVID-19 vaccines for low- and middle-income countries. Transactions of The Royal Society of Tropical Medicine and Hygiene. 2021;115(5):447-456. doi:10.1093/trstmh/trab045

5. Ebinger JE, Fert-Bober J, Printsev I, et al. Antibody responses to the BNT162b2 mRNA vaccine in individuals previously infected with SARS-CoV-2. Nat Med. 2021;27(6):981-984. doi:10.1038/s41591-02101325-6

6. Callaway E. Mix-and-match COVID vaccines trigger potent immune response. Nature. 2021;593(7860):491. doi:10.1038/d41586-021-01359-3

7. Ramasamy MN, Minassian AM, Ewer KJ, et al. Safety and immunogenicity of ChAdOx1 nCoV-19 vaccine administered in a prime-boost regimen in young and old adults (COV002): a single-blind, randomised, controlled, phase 2/3 trial. Lancet. 2020;396(10267):1979-1993. doi:10.1016/S0140-6736(20)32466-1
8. Voysey M, Clemens SAC, Madhi SA, et al. Safety and efficacy of the ChAdOx1 nCoV-19 vaccine (AZD1222) against SARS-CoV-2: an interim analysis of four randomised controlled trials in Brazil, South Africa, and the UK. Lancet. 2021;397(10269):99-111. doi:10.1016/S0140-6736(20)32661-1

9. AstraZeneca. COVID-19 vaccine AstraZeneca real-world evidence summary; August, 2021:38-39. Available from: https://www.astraze neca.com/content/dam/az/covid-19/media/factsheets/COVID-19 Vaccine_AstraZeneca_Real-World_Evidence_Summary.pdf. Accessed November 16, 2021.

10. World Health Organization: WHO. Bangladesh COVID-19 reported to WHO [Internet]; 2021. Available from: https://covid19.who.int/ region/searo/country/bd. Accessed November 16, 2021.

11. Callegaro A, Borleri D, Farina C, et al. Antibody response to SARS-CoV2 vaccination is extremely vivacious in subjects with previous SARSCoV-2 infection. J Med Virol. 2021;93(7):4612-4615. doi:10.1002/ jmv.26982

12. Folegatti PM, Ewer KJ, Aley PK, et al. Safety and immunogenicity of the ChAdOx1 $\mathrm{nCoV}-19$ vaccine against SARS-CoV-2: a preliminary report of a phase 1/2, single-blind, randomised controlled trial. Lancet. 2020;396(10249):467-478. doi:10.1016/S0140-6736(20)31604-4

13. Ewer KJ, Barrett JR, Belij-Rammerstorfer S, et al. T cell and antibody responses induced by a single dose of ChAdOx $1 \mathrm{nCoV}-19$ (AZD1222) vaccine in a phase 1/2 clinical trial. Nat Med. 2021;27 (2):270-278. doi:10.1038/s41591-020-01194-5

14. Anderson EJ, Rouphael NG, Widge AT, et al. Safety and immunogenicity of SARS-CoV-2 mRNA-1273 vaccine in older adults. $N$ Engl $J$ Med. 2020;383(25):2427-2438. doi:10.1056/NEJMoa2028436

15. Pottegard A, Lund LC, Karlstad Ø, et al. Arterial events, venous thromboembolism, thrombocytopenia, and bleeding after vaccination with Oxford-AstraZeneca ChAdOx1-S in Denmark and Norway: population based cohort study. $B M J$. 2021;373. doi:10.1136/bmj.n1114
Infection and Drug Resistance

\section{Publish your work in this journal}

Infection and Drug Resistance is an international, peer-reviewed openaccess journal that focuses on the optimal treatment of infection (bacterial, fungal and viral) and the development and institution of preventive strategies to minimize the development and spread of resistance. The journal is specifically concerned with the epidemiology of antibiotic resistance and the mechanisms of resistance development and diffusion in both hospitals and the community. The manuscript management system is completely online and includes a very quick and fair peerreview system, which is all easy to use. Visit http://www.dovepress.com/ testimonials.php to read real quotes from published authors. 